\title{
Band structure of MoSTe Janus nanotubes
}

\section{Guldberg Mikkelsen, August Edwards; Bölle, Felix Tim; Thygesen, Kristian Sommer; Vegge, Tejs; Castelli, Ivano Eligio}

\section{Published in:}

Physical Review Materials

Link to article, DOI:

10.1103/PhysRevMaterials.5.014002

Publication date:

2021

Document Version

Publisher's PDF, also known as Version of record

Link back to DTU Orbit

Citation (APA):

Guldberg Mikkelsen, A. E., Bölle, F. T., Thygesen, K. S., Vegge, T., \& Castelli, I. E. (2021). Band structure of MoSTe Janus nanotubes. Physical Review Materials, 5(1), [014002].

https://doi.org/10.1103/PhysRevMaterials.5.014002

\section{General rights}

Copyright and moral rights for the publications made accessible in the public portal are retained by the authors and/or other copyright owners and it is a condition of accessing publications that users recognise and abide by the legal requirements associated with these rights.

- Users may download and print one copy of any publication from the public portal for the purpose of private study or research.

- You may not further distribute the material or use it for any profit-making activity or commercial gain

- You may freely distribute the URL identifying the publication in the public portal 


\title{
Band structure of MoSTe Janus nanotubes
}

\author{
August E. G. Mikkelsen, ${ }^{1}$ Felix T. Bölle $\odot,{ }^{1}$ Kristian S. Thygesen, ${ }^{2}$ Tejs Vegge $\odot,{ }^{1}$ and Ivano E. Castelli $\odot^{1, *}$ \\ ${ }^{1}$ Department of Energy Conversion and Storage, Department of Physics, Technical University of Denmark, \\ DK-2800 Kongens Lyngby, Denmark \\ ${ }^{2}$ CAMD, Department of Physics, Technical University of Denmark, DK-2800 Kongens Lyngby, Denmark
}

(Received 23 September 2020; revised 1 December 2020; accepted 16 December 2020; published 12 January 2021)

\begin{abstract}
Nanotubes generated by rolling up transition metal dichalcogenide Janus monolayers are a new class of low-dimensional materials, which are expected to display unique electronic properties compared to their parent two- and three-dimensional structures. Here, we investigate the band structure of $1 H$-MoSTe Janus armchair and zigzag nanotubes, which were recently hypothesized to be stable as single-walled structures with radii of only a few nanometers. We first investigate the most stable nanotube sizes and assess the influence of quantum confinement and curvature on the band structures, showing that these are heavily modified by curvature while confinement effects are negligible. The curvature dependence is then further studied by analyzing the band gap dependence on the nanotube radius, where band gap changes as large as $0.5 \mathrm{eV}$ are observed. By investigating the band edge positions and orbital projected density of states for different tube sizes, we find that this high sensitivity is mainly attributed to the Mo $d$ states in the conduction band.
\end{abstract}

DOI: 10.1103/PhysRevMaterials.5.014002

\section{INTRODUCTION}

During the past decades, the study and characterization of low-dimensional materials has been one of the most active fields in materials science. The immense scientific interest is fueled by the expectation that low-dimensional materials will have novel applications within a broad range of research areas, from batteries and electrocatalysis, to electronics and photonics [1-3]. On the experimental side this has resulted in numerous reports of novel two- and one-dimensional (2D and 1D) structures [4-9], and on the computational side several databases have emerged containing thousands of hypothetical materials with remarkably different properties compared to their parent 3D structures [10-12].

A particular class of low-dimensional materials, which have been studied intensely, are 2D monolayers based on transition metal dichalcogenides (TMDs). TMD monolayers have the chemical formula $M X_{2}$, where $M$ is a transition metal atom and $X$ is a chalcogen atom, and include examples such as $\mathrm{MoS}_{2}, \mathrm{MoTe}_{2}$, and $\mathrm{WS}_{2}$, all of which have been synthesized experimentally [6,13,14]. Furthermore, several TMDs have also been synthesized in a one-dimensional form as multiwalled nanotubes with diameters of several nanometers $[8,9]$. Such tubular structures are expected to have promising applications within a broad range of scientific areas such as, e.g., gas separation and capture, catalysis, solid lubrication, and controlled drug delivery [15], but so far advancements have been hampered by difficulties in producing single-wall nanotubes with a well-defined radius.

A less known class of 2D structures are the so-called TMD Janus monolayers with the chemical formula $M X Y$

\footnotetext{
*Corresponding author: ivca@dtu.dk
}

[16]. Such layers can, at least in principle, be obtained by substitution of the chalcogen atoms on one side of a regular $M X_{2}$ monolayer, and examples include MoSSe, MoSTe, and WSSe, among which MoSSe was recently synthesized [17]. TMD Janus monolayers are particularly interesting because their asymmetry gives rise to an intrinsic strain, which makes self-rolling into the nanotube structure energetically favorable $[18,19]$. In contrast to the multiwalled nanotubes mentioned above, such nanotubes are expected to be stable as single-walled structures with diameters of only a few nanometers. At such small radii the finite size and curvature will subject the atoms to significant distortion, and these nanotubes are thus expected to display unique and tunable electronic properties compared to their parent monolayers [20-22].

We have recently conducted a comprehensive study on the thermodynamic stability of a wide range of inorganic nanotubes obtained from the wrapping of Janus monolayers [23]. As part of this effort, the present paper is devoted to a more detailed study on the electronic properties of nanotubes made from monolayer MoSTe in the $1 H$ phase, which was recently proposed as a promising candidate material for piezoelectric applications [24].

A sketch of how the relevant MoSTe nanotubes are constructed is shown in Fig. 1(a): Starting from a $1 H$ monolayer of MoSTe described by the hexagonal unit cell vectors $\mathbf{a}_{1}, \mathbf{a}_{2}$, a change of basis to the symmetrically equivalent rectangular basis described by the vectors $\mathbf{a}_{1}^{\prime}, \mathbf{a}_{2}^{\prime}$ is first performed. Armchair and zigzag MoSTe nanotubes of different sizes are then obtained by wrapping up nanoribbons corresponding to different numbers of unit cell repetitions along either $\mathbf{a}_{1}^{\prime}$ (zigzag) or $\mathbf{a}_{2}^{\prime}$ (armchair). In the screening study of Ref. [23], both armchair and zigzag type nanotubes were investigated (chiral nanotubes were not considered), and both types were found to 
(a) 2D MosTe

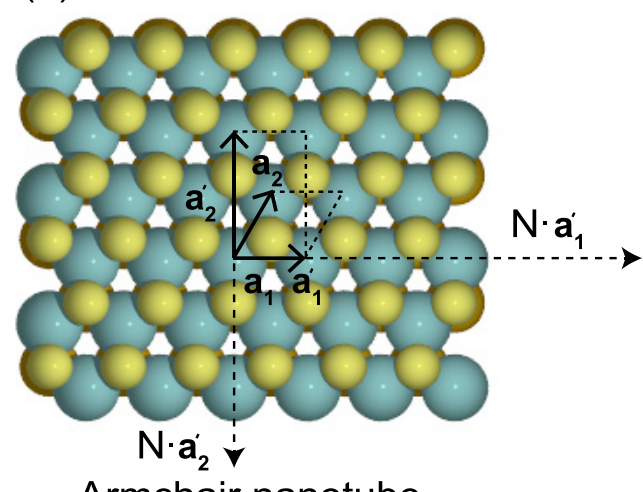

Armchair nanotube

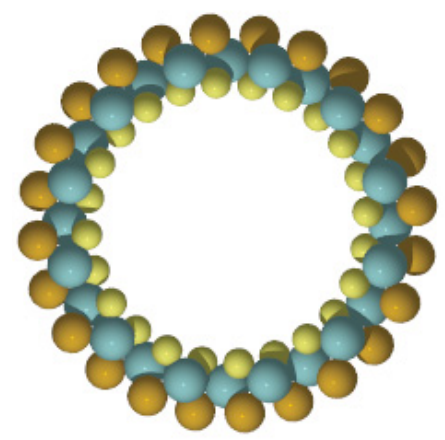

Zigzag nanotube

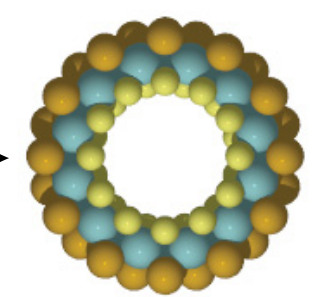

Brillouin zones

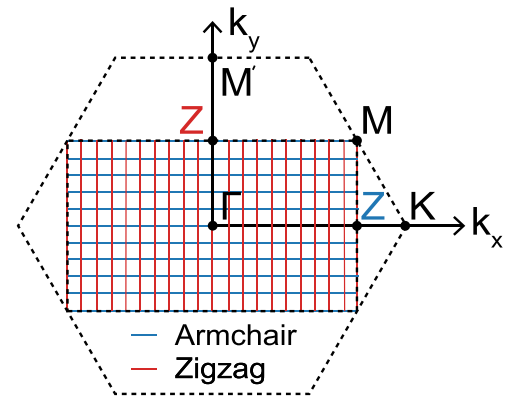

(b)

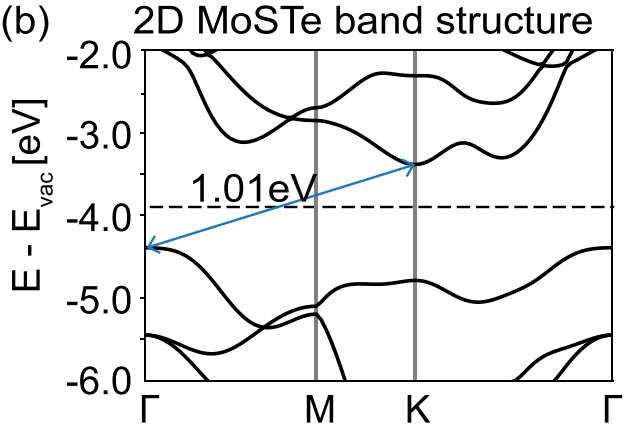

(c)

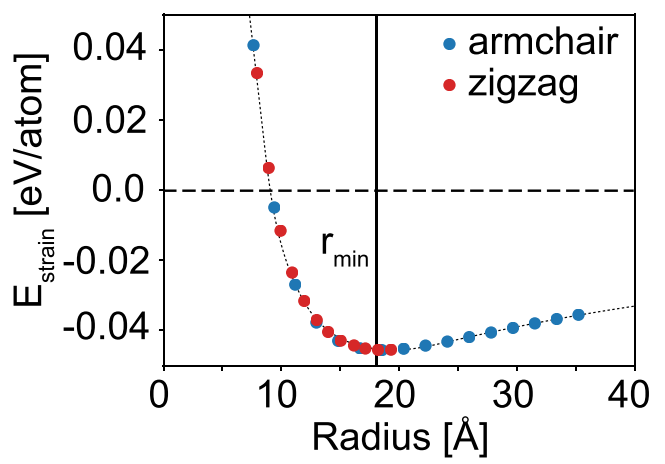

FIG. 1. (a) Sketch of how armchair and zigzag nanotubes are constructed by rolling up a 2D MoSTe monolayer along different directions. The different unit cells involved are indicated and their relation in reciprocal space is displayed in the top right. (b) Band structure of a $2 \mathrm{D}$ MoSTe monolayer with the band gap indicated. (c) Calculated strain energies of armchair and zigzag MoSTe nanotubes for different radii. A minimum strain energy is attained at $r_{\min }$ which is the most stable nanotube radius.

show promising signs of thermodynamic stability compared to both their bulk constituents as well as their parent 2D monolayer. The latter is demonstrated in Fig. 1(c), which displays the nanotube strain energy, defined as the energy difference with respect to the parent monolayer, as a function of the radius. Evidently both armchair and zigzag MoSTe nanotubes are stable with respect to their parent 2D layer with a peak in stability at a radius of only $\sim 18 \AA$. Combining this with the fact that the dynamical stability of both $1 H$-MoSTe monolayers and MoSTe nanotubes has been confirmed through phonon calculations [19,24], makes these structures particularly well suited for a more detailed investigation focusing on the electronic properties.

The paper is structured as follows: Starting from the most stable MoSTe zigzag and armchair configurations shown in Fig. 1(c), we first analyze the band structures and how they differ from their parent monolayer as well as the symmetric $\mathrm{MoS}_{2}$ and $\mathrm{MoTe}_{2}$ structures. We then proceed to perform a more systematic investigation of how the band structure, and in particular the band gap, depends on the size of the simulated nanotubes, and how the behavior may be qualitatively understood from the orbital projected density of states (PDOS) around the Fermi level. Our study demonstrates how 1D Janus TMD tubes can display remarkably different electronic properties compared to their $2 \mathrm{D}$ and $3 \mathrm{D}$ counterparts, thus showing that targeted screening of such nanotubes for specific applications such as catalysis and thermoelectrics might be of future interest.

\section{COMPUTATIONAL METHODOLOGY}

All calculations are carried out using the Perdew-BurkeErnzerhof (PBE) functional [25] as implemented in the Vienna $a b$ initio simulation package [26]. We employ a plane-wave basis set with an energy cutoff of $550 \mathrm{eV}$. The construction and relaxation of the nanotubes is performed using the atomistic simulation environment (ASE) [27] and follows the procedure outlined in Ref. [23], where a $k$-point density $>4.7$ per $\AA^{-1}$ is used to sample the Brillouin zone and the forces on the atoms are converged to less than 0.02 $\mathrm{eV} / \AA$. To obtain well-converged band structures and band gaps we perform self-consistent calculations for the relaxed structures using a gamma-centered uniform $k$ mesh with a density greater than 15.0 per $\AA^{-1}$. We use a dipole correction for all structures with an out-of-plane dipole moment such as the nanoribbons described in Sec. III. For the band structure, band edge, and projected density of states results presented in Figs. 2 and 3 we have used the vacuum level energy defined by the asymptotic value of the Hartree potential as our reference. For a nanotube we obtain this by averaging the Hartree potential on a radial grid centered on each nanotube. For the asymmetric nanoribbons, where a dipole correction is applied, we use the asymptotic value of the planar averaged Hartree potential on the side of the structure, which would make up the outer side of the corresponding nanotube. All the calculations were performed with a computational workflow, which is described in detail in Ref. [23], and may be accessed via 

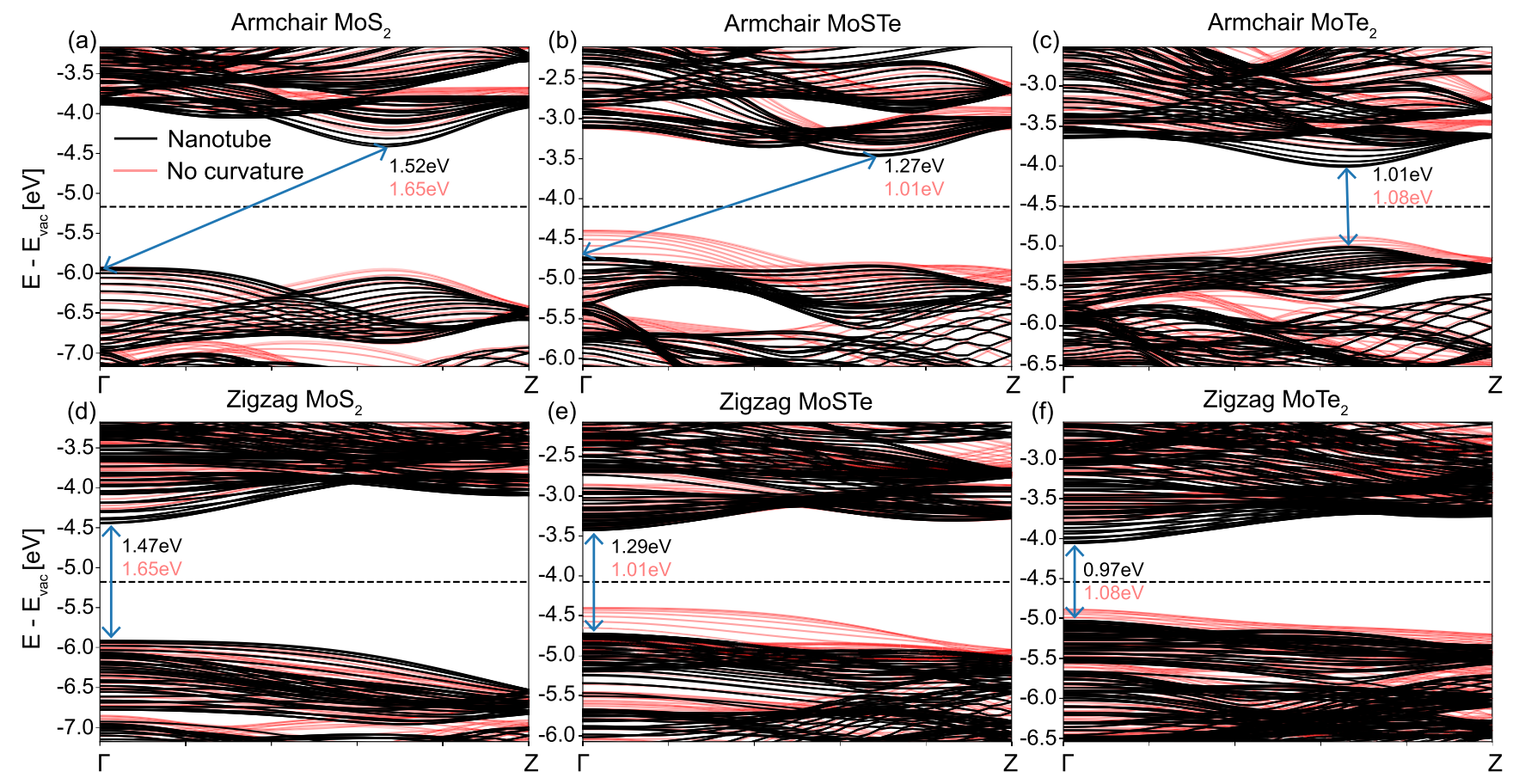

FIG. 2. Band structures of armchair and zigzag $\mathrm{MoS}_{2}$, MoSTe and $\mathrm{MoTe}_{2}$ nanotubes (solid black lines) along with the corresponding curvatureless band structures (opaque red lines). The band gap transitions are indicated by blue arrows. (a) $\mathrm{MoS}_{2}$ armchair nanotube with a radius of $\sim 16 \AA$. (b) MoSTe armchair nanotube with a radius of $\sim 16.5 \AA$. (c) Armchair MoTe ${ }_{2}$ nanotube with a radius of $\sim 18 \AA$. (d) Zigzag $\mathrm{MoS}_{2}$ nanotube with a radius of $\sim 15 \AA$. (e) Zigzag MoSTe nanotube with a radius of $\sim 16 \AA$. (f) Zigzag MoTe $\mathrm{Z}_{2}$ nanotube with a radius of $\sim 15 \AA$.

Ref. [28]. The relaxed 2D sheets and nanotube structures are furthermore stored in a database in the DTU Data Repository [29].

\section{RESULTS AND DISCUSSION}

Figure 2 displays the band structure for selected armchair [Figs. 2(a)-2(c)] and zigzag [Figs. 2(d)-2(f)] $\mathrm{MoS}_{2}, \mathrm{MoSTe}$, and $\mathrm{MoTe}_{2}$ nanotubes. The nanotubes have been selected to correspond to the most stable radii for the MoSTe armchair and zigzag configurations as shown in Fig. 1(c). Consequently, the MoSTe armchair nanotube was chosen to have a radius of $\sim 18.4 \AA$ and 120 atoms in the unit cell, while for the zigzag nanotube a radius of $\sim 18.1 \AA$ and 204 atoms in the unit cell was used. The sizes of the symmetric $\mathrm{MoS}_{2}$ and $\mathrm{MoTe}_{2}$ nanotubes were chosen to be consistent with these numbers, i.e., with 120 and 204 atoms in the unit cell for the armchair and zigzag, respectively. We emphasize that these nanotubes, in contrast to MoSTe, are not formed from a Janus structure and are therefore likely to be unstable with respect to their planar counterpart.

To understand the general features of the nanotube band structures in Fig. 2, we first note that these are influenced by two separate structural effects: (i) The effect of quantum confinement arising from the finite extent of a nanotube along its wrapping axis. This is manifested by periodicity of the electronic Bloch states along its circumference as discussed below. (ii) The effect of curvature arising from the bending of the parent nanoribbon into the nanotube form.

In order to separate the effects described in (i) and (ii) we have included in Fig. 2 a set of band structures, which neglect the effect of the nanotube curvature. These are obtained from the 2D band structure of the parent MoSTe monolayer [see Fig. 2(b)] following the procedure described in Ref. [30], where the $k$ points inside the Brillouin zone allowed by (i) are selected and used to generate the corresponding 1D band structure. As an example of how this is done, we consider generating the curvatureless band structure equivalent of a nanotube corresponding to $N$ repetitions along the armchair direction as shown in Fig. 1(a). In this case, periodicity of the electronic states along the wrapping direction combined with Bloch's theorem yields $\psi(\mathbf{R})=\psi\left(\mathbf{R}+N \cdot \mathbf{a}_{2}^{\prime}\right) \Rightarrow \psi(\mathbf{R})=$ $\psi(\mathbf{R}) e^{i N \mathbf{k} \cdot \mathbf{a}_{2}^{\prime}}$, which leads to the following quantization condition for the allowed values of $\mathbf{k}$ :

$$
\mathbf{k} \cdot \mathbf{a}_{2}^{\prime}=2 \pi \frac{m}{N}, \quad m=0,1, \ldots, N-1 .
$$

The result is a discrete set of allowed $k$ points along the $\mathbf{a}_{2}^{\prime}$ direction, which, as shown in Fig. 1(a), is along the $\Gamma-M^{\prime}$ path in reciprocal space. The band structure is then obtained from the 2D band structure of MoSTe by plotting its value for each discrete $k$ value in Eq. (1) in the direction perpendicular to $\Gamma-M^{\prime}$ as shown by the blue lines in Fig. 1(a). A similar analysis for the zigzag direction leads to a discrete set of $k$ values along the $\Gamma-K$ path and in this case the band structure is obtained by plotting the values of the 2D band structure along the path marked by the red lines of Fig. 1(a). As shown in Fig. 1(a), we label the boundary of the rectangular Brillouin zone as $Z$ in both the armchair and zigzag directions to be consistent with the labeling of the $Z$ point in the nanotube band structures of Fig. 2. 

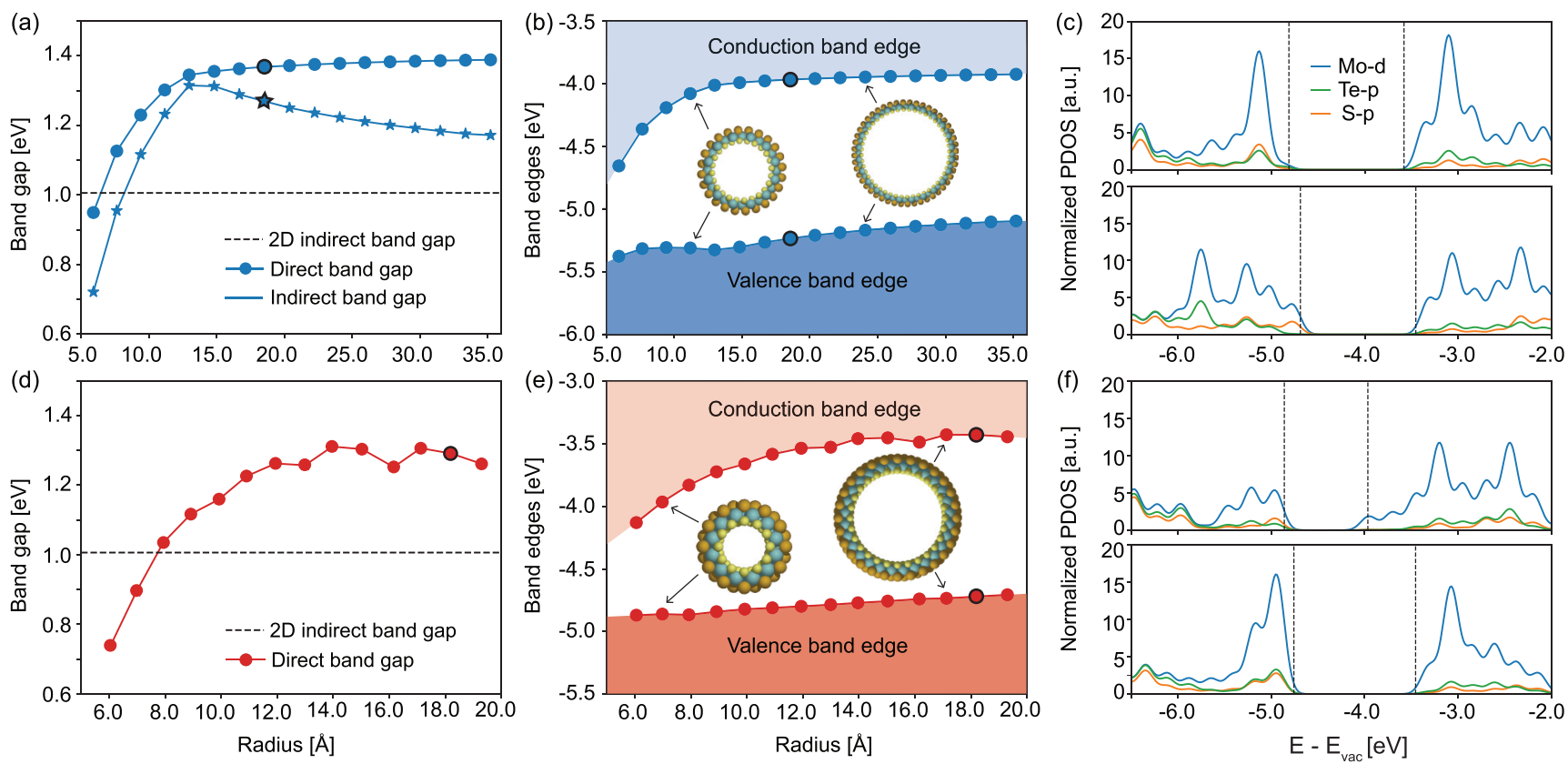

FIG. 3. (a) Band gap dependence on the radius for MoSTe armchair nanotubes with the most stable tube radius highlighted. Both indirect and direct band gaps are shown, and the indirect band gap of 2D MoSTe is indicated as the dashed black line. (b) Position of the valence and conduction band edges for MoSTe armchair nanotubes as a function of the radius. An illustration of the two nanotubes used for the corresponding PDOS plots is also included. (c) PDOS in a region around the Fermi level for an MoSTe armchair nanotube with radius $\sim 11 \AA$ (top) and for an MoSTe armchair nanotube with radius $22 \AA$ (bottom). (d) Band gap dependence on the radius for MoSTe zigzag nanotubes with the most stable tube radius highlighted and with the indirect band gap of 2D MoSTe indicated by the black dashed line. The band gap is direct for all plotted radii. (e) Position of the valence and conduction band edges for MoSTe zigzag nanotubes as a function of the radius. An illustration of the two nanotubes used for the corresponding PDOS plots is also included. (f) PDOS around the Fermi level for an MoSTe zigzag nanotube with radius $\sim 7 \AA$ (top) and for an MoSTe armchair nanotube with radius $\sim 12 \AA$ (bottom).

Comparing the band structures of Fig. 2, we can make several qualitative observations. First of all, the band structures of the Janus MoSTe nanotubes appear significantly different from those of the symmetric $\mathrm{MoS}_{2}$ and $\mathrm{MoTe}_{2}$ nanotubes. Second, we observe that while the nanotube and nanoribbon band structures share the same overall features, the nanotube band gaps are heavily modified by curvature. Indeed, by comparing Figs. 2(b) and 2(e) with the band structure of 2D MoSTe shown in Fig. 1(b), we can see that the nanotube band gaps are significantly different from the band gap of 2D MoSTe, while the nanoribbon band gaps practically coincide with the latter. This shows that quantization has a very minor influence on the size of the band gap. To understand this we first note that the band gap of 2D MoSTe corresponds to an indirect $\Gamma-K$ transition. Since $\mathbf{k}=\mathbf{0}$ is included in the discrete set of $k$ values in Eq. (1) for all values of $N$, the $\Gamma$ point will likewise be included when generating the nanoribbon band structure for a nanotube of any size. Any dependence of the band gap must thus be attributed to whether or not the discrete set of $k$ points of Eq. (1) includes the $K$ point. For armchair nanotubes, this will always be included since the discretization is along $\Gamma-M^{\prime}$. Consequently, their band gap cannot be influenced by confinement effects. For zigzag nanotubes this is a possibility, but for the typical tube sizes simulated, the $k$ mesh in Eq. (1) will be practically dense and any dependence is extremely minor. We thus conclude that the band gap changes observed in Fig. 2 are purely an effect of the curvature induced by the bending of the atoms in the nanotube form.

To explore further the effect of curvature on the band gap, we have calculated the band gap for MoSTe armchair and zigzag nanotubes of different radii as displayed in Figs. 3(a) and 3(d). From this we can see that the band gap depends strongly on the radius of the simulated nanotubes and is seen to increase from a value below the band gap of 2D MoSTe to a value above, followed by a slow decay towards this limiting value. This behavior, which is in agreement with the results reported in Ref. [19], may be qualitatively understood from Figs. 3(b) and 3(e), which display the position of the valence and conduction band edge as a function of the radius of the simulated nanotubes. Evidently, the size dependence of the band gap is mainly attributed to the position of the conduction band edge, which, compared to the valence band edge, increases sharply as a function of the radius. Based on this, it is therefore natural to investigate the PDOS around the conduction band edge to determine what states are mainly responsible for the observed band gap sensitivity. The PDOS for selected armchair and zigzag nanotubes is shown in Figs. 3(c) and 3(f), and from these plots we see that the lower part of the conduction band is mainly composed of states with Mo $d$ character. We therefore conjecture that these states are particularly sensitive to the nanotube curvature, and a natural starting point for future work would therefore be to try and understand the origin of this sensitivity and whether or not 
similar trends will be observed for Janus nanotubes based on other elements.

Another natural question to ask is whether the conclusions reported here hold when employing higher-level electronic structure methods and/or when including spin-orbit coupling. The latter is in fact known to heavily modify the electronic structure of many TMD monolayers [24,31] and is therefore particularly relevant. While not reported here, we have in fact performed band structure calculations for selected nanotube sizes with spin-orbit coupling included and found that while it does indeed lead to a modest change in the band gap of $\sim 50 \mathrm{meV}$, this acts mostly as a constant shift across different nanotube radii. The conclusions reported in Fig. 3 are thus unchanged by the inclusion of spin-orbit coupling.

\section{CONCLUSION}

We have investigated the band structure of armchair and zigzag $1 H$-MoSTe Janus nanotubes with special emphasis on understanding how these differ from that of their parent monolayer. By comparing the band structures of the most stable MoSTe tube size with those of the symmetric $\mathrm{MoS}_{2}$ and $\mathrm{MoTe}_{2}$ nabotubes and nanoribbons, we were able to separate the effects of quantum confinement and curvature, and we found that confinement effects were modest while curvature led to large changes in the band gap. We further analyzed the band gap changes by studying its dependence of the nanotube radius and found variations in the band gap of up to $0.5 \mathrm{eV}$ with values both above and below that of $2 \mathrm{D}$ monolayer MoSTe. By investigating the position of the band edges and PDOS for different tube sizes, we found that these large variations are mainly attributed to the Mo conduction band $d$ states.

Our study demonstrates that Janus TMD nanotubes can display remarkably different and tunable electronic properties compared to their parent 2D monolayers, which make them an interesting platform for computational materials screening targeting specific applications within materials science.

\section{ACKNOWLEDGMENTS}

A.E.G.M., F.T.B., T.V., and I.E.C. acknowledge support from the Department of Energy Conversion and Storage, Technical University of Denmark, through the Special Competence Initiative Autonomous Materials Discovery [32]. K.S.T. acknowledges support from the European Research Council (ERC) under the European Union's Horizon 2020 research and innovation programme (Grant Agreement No. 773122, LIMA).
[1] A. C. Ferrari, F. Bonaccorso, V. Fal'ko, K. S. Novoselov, S. Roche, P. Bøggild, S. Borini, F. H. L. Koppens, V. Palermo, N. Pugno, J. A. Garrido, R. Sordan, A. Bianco, L. Ballerini, M. Prato, E. Lidorikis, J. Kivioja, C. Marinelli, T. Ryhänen, A. Morpurgo et al., Nanoscale 7, 4598 (2015).

[2] G. R. Bhimanapati, Z. Lin, V. Meunier, Y. Jung, J. Cha, S. Das, D. Xiao, Y. Son, M. S. Strano, V. R. Cooper, L. Liang, S. G. Louie, E. Ringe, W. Zhou, S. S. Kim, R. R. Naik, B. G. Sumpter, H. Terrones, F. Xia, Y. Wang et al., ACS Nano 9, 11509 (2015).

[3] Q. Deng, J. Zhao, T. Wu, G. Chen, H. A. Hansen, and T. Vegge, J. Catal. 370, 378 (2019).

[4] K. S. Novoselov, Science 306, 666 (2004).

[5] S. Iijima and T. Ichihashi, Nature (London) 363, 603 (1993).

[6] K. F. Mak, C. Lee, J. Hone, J. Shan, and T. F. Heinz, Phys. Rev. Lett. 105, 136805 (2010).

[7] L. Ci, L. Song, C. Jin, D. Jariwala, D. Wu, Y. Li, A. Srivastava, Z. F. Wang, K. Storr, L. Balicas, F. Liu, and P. M. Ajayan, Nat. Mater. 9, 430 (2010).

[8] R. Tenne, L. Margulis, M. Genut, and G. Hodes, Nature (London) 360, 444 (1992).

[9] R. Tenne, M. Homyonfer, and Y. Feldman, Chem. Mater. 10, 3225 (1998).

[10] S. Haastrup, M. Strange, M. Pandey, T. Deilmann, P. S. Schmidt, N. F. Hinsche, M. N. Gjerding, D. Torelli, P. M. Larsen, A. C. Riis-Jensen, J. Gath, K. W. Jacobsen, J. J. Mortensen, T. Olsen, and K. S. Thygesen, 2D Mater. 5, 042002 (2018).

[11] N. Mounet, M. Gibertini, P. Schwaller, D. Campi, A. Merkys, A. Marrazzo, T. Sohier, I. Castelli, A. Cepellotti, G. Pizzi, and N. Marzari, Nat. Nanotechnol. 13, 246 (2018).

[12] H. L. Zhuang, A. K. Singh, and R. G. Hennig, Phys. Rev. B 87, 165415 (2013).
[13] C. H. Naylor, W. M. Parkin, J. Ping, Z. Gao, Y. R. Zhou, Y. Kim, F. Streller, R. W. Carpick, A. M. Rappe, M. Drndić, J. M. Kikkawa, and A. T. C. Johnson, Nano Lett. 16, 4297 (2016).

[14] M. Okada, T. Sawazaki, K. Watanabe, T. Taniguch, H. Hibino, H. Shinohara, and R. Kitaura, ACS Nano 8, 8273 (2014).

[15] M. Serra, R. Arenal, and R. Tenne, Nanoscale 11, 8073 (2019).

[16] A. C. Riis-Jensen, T. Deilmann, T. Olsen, and K. S. Thygesen, ACS Nano 13, 13354 (2019).

[17] A.-Y. Lu, H. Zhu, J. Xiao, C.-P. Chuu, Y. Han, M.-H. Chiu, C.-C. Cheng, C.-W. Yang, K.-H. Wei, Y. Yang, Y. Wang, D. Sokaras, D. Nordlund, P. Yang, D. A. Muller, M.-Y. Chou, X. Zhang, and L.-J. Li, Nat. Nanotechnol. 12, 744 (2017).

[18] Q.-1. Xiong, J. Zhou, J. Zhang, T. Kitamura, and Z.-h. Li, Phys. Chem. Chem. Phys. 20, 20988 (2018).

[19] W. Zhao, Y. Li, W. Duan, and F. Ding, Nanoscale 7, 13586 (2015).

[20] Y. F. Luo, Y. Pang, M. Tang, Q. Song, and M. Wang, Comput. Mater. Sci. 156, 315 (2019).

[21] R. Evarestov, A. Kovalenko, and A. Bandura, Physica E 115, 113681 (2020).

[22] S. Oshima, M. Toyoda, and S. Saito, Phys. Rev. Materials 4, 026004 (2020).

[23] F. T. Bölle, A. E. G. Mikkelsen, K. S. Thygesen, T. Vegge, and I. E. Castelli, arXiv:2011.14708.

[24] M. Yagmurcukardes, C. Sevik, and F. M. Peeters, Phys. Rev. B 100, 045415 (2019).

[25] J. P. Perdew, K. Burke, and M. Ernzerhof, Phys. Rev. Lett. 77, 3865 (1996).

[26] G. Kresse and D. Joubert, Phys. Rev. B 59, 1758 (1999).

[27] A. H. Larsen, J. J. Mortensen, J. Blomqvist, I. E. Castelli, R. Christensen, M. Dułak, J. Friis, M. N. Groves, B. Hammer, C. Hargus, E. D. Hermes, P. C. Jennings, P. B. Jensen, 
J. Kermode, J. R. Kitchin, E. L. Kolsbjerg, J. Kubal, K. Kaasbjerg, S. Lysgaard, J. B. Maronsson et al., J. Phys.: Condens. Matter 29, 273002 (2017).

[28] https://gitlab.com/asc-dtu/workflows/nanotubes/.

[29] DTU Data Repository, https://doi.org/10.11583/DTU. 13469244.
[30] N. Hamada, S.-i. Sawada, and A. Oshiyama, Phys. Rev. Lett. 68, 1579 (1992).

[31] Z. Y. Zhu, Y. C. Cheng, and U. Schwingenschlögl, Phys. Rev. B 84, 153402 (2011).

[32] AiMade (Autonomous Materials Discovery), http://aimade. org/. 